\title{
Squeezing and photon distribution in a vibrating cavity
}

\author{
V V Dodonovf and M A Andreata \\ Departamento de Física, Universidade Federal de São Carlos, \\ Via Washington Luiz km 235, 13565-905 São Carlos, SP, Brazil
}

\begin{abstract}
We obtain explicit analytical expressions for the quadrature variances and the photon distribution functions of the electromagnetic field modes excited from vacuum or thermal states due to the non-stationary Casimir effect in an ideal one-dimensional Fabry-Perot cavity with vibrating walls, provided the frequency of vibrations is close to a multiple frequency of the fundamental unperturbed electromagnetic mode.
\end{abstract}

PACS numbers: 42.50.Lc, 03.70.+k, 03.65.Bz

Submitted to: J. Phys. A: Math. Gen.

$\ddagger$ E-mail: vdodonov@power.ufscar.br 


\section{Introduction}

During last decade, the attention of many authors was attracted to quantum phenomena in cavities and media with moving boundaries, known under the names non-stationary Casimir effect (NSCE) [1], dynamical Casimir effect [2], or mirror (motion) induced radiation [3, 4. For the most recent achievements in this field and references to other works see, e.g. [5-23] (the problems of the classical electrodynamics with moving boundaries were studied, e.g. in [24, 25]). A complete analytical solution to the problem of a one-dimensional (Fabry-Perot) ideal cavity with resonantly vibrating boundaries was found recently in [17]. This solution holds for any moment of time (provided the amplitude of the wall vibrations is small enough); moreover, it enables not only to calculate the number of photons created from an arbitrary initial state, but also to account for the effects of detuning from a strict resonance.

In the present article, continuing the line of research of [17], we calculate the effects of squeezing and find the photon distribution function of the field in each mode inside the cavity. Although a possibility of squeezing the electromagnetic field in a cavity with moving boundaries due to the NSCE was discussed for the first time ten years ago [26], the concrete calculations of the variances of the field quadrature components were made only in the short-time [26, 27] and long-time [28, 29] limits under the condition of the strict resonance. As to the photon statistics in an oscillating Fabry-Perot cavity, it was not discussed at all until now (the photon distribution in a 3D nondegenerate resonantly driven cavity was found in [5]).

\section{Field operator in a $1 \mathrm{D}$ cavity with oscillating boundaries}

We start with a brief description of the main results of 17 which are used in the subsequent sections. Following the model of a "scalar electrodynamics" [30, we assume that a $1 \mathrm{D}$ cavity is formed by two infinite ideal plates whose positions are given by $x_{l e f t} \equiv 0$ and

$$
x_{\text {right }} \equiv L(t)=L_{0}\left(1+\varepsilon \sin \left[p \omega_{1}(1+\delta) t\right]\right), \quad|\varepsilon| \ll 1, \quad|\delta| \ll 1
$$

where $\omega_{1}=\pi c / L_{0}$ and $p=1,2, \ldots$ As was shown in [17, the results can be easily generalized to a generic case when both the mirrors oscillate (see also [11, 22]). We are looking for the only component of the operator vector potential of the electromagnetic field $\hat{A}(x, t)$ in the Heisenberg representation in the form (hereafter $c=\hbar=1$ )

$$
\hat{A}(x, t)=\sum_{n=1}^{\infty} \frac{2}{\sqrt{n}}\left[\hat{b}_{n} \psi^{(n)}(x, t)+\text { h.c. }\right], \quad\left[\hat{b}_{n}, \hat{b}_{k}^{\dagger}\right]=\delta_{n k} .
$$

This operator must satisfy the wave equation $\hat{A}_{t t}-\hat{A}_{x x}=0$ and the boundary conditions 30 $\hat{A}(0, t)=\hat{A}(L(t), t)=0$. For $t<0$, when the wall is assumed to be at rest, function $\psi^{(n)}(x, t)$ has a simple factorized form $\psi_{0}^{(n)}(x, t)=e^{-i \omega_{n} t} \sin \left(\pi n x / L_{0}\right), \omega_{n}=n \omega_{1}$. The normalization factors $2 / \sqrt{n}$ in (2.2) are chosen in such a way that the energy of the 
field in the stationary case can be represented as a sum of energies of independent mode oscillators (see the next section).

For $t \geq 0$, following [31] we expand each function $\psi^{(n)}(x, t)$ in a series with respect to the instantaneous basis

$$
\psi^{(n)}(x, t)=\sqrt{\frac{L_{0}}{L(t)}} \sum_{k=1}^{\infty} Q_{k}^{(n)}(t) \sin \left[\frac{\pi k x}{L(t)}\right] .
$$

This way we automatically satisfy the boundary conditions. Then the wave equation is replaced by the infinite set of coupled differential equations

$$
\ddot{Q}_{k}^{(n)}+\omega_{k}^{2}(t) Q_{k}^{(n)}=2 \sum_{j=1}^{\infty} g_{k j}(t) \dot{Q}_{j}^{(n)}+\sum_{j=1}^{\infty} \dot{g}_{k j}(t) Q_{j}^{(n)}+\mathcal{O}\left(g_{k j}^{2}\right),
$$

where

$$
\omega_{k}(t)=k \pi / L(t), \quad g_{k j}=-g_{j k}=(-1)^{k-j} \frac{2 k j \dot{L}(t)}{\left(j^{2}-k^{2}\right) L(t)} .
$$

As was shown in [17], the set of equations (2.4) can be significantly simplified in the case of the (quasi)resonance oscillations of the wall given by the dependence (2.1), if one writes

$$
Q_{k}^{(n)}(t)=\rho_{k}^{(n)} e^{-i \omega_{k}(1+\delta) t}-\rho_{-k}^{(n)} e^{i \omega_{k}(1+\delta) t}
$$

assuming the coefficients $\rho_{k}^{(n)}(k= \pm 1, \pm 2, \ldots ; n=1,2, \ldots)$ to be slowly varying functions of time, whose derivatives are proportional to the small parameter $\varepsilon$. Putting (2.5) into equation (2.4) and neglecting the second order terms like $\ddot{\rho}_{k}^{(n)} \sim \varepsilon^{2}$, one obtains, after averaging over fast oscillations with the multiple frequencies of $\omega_{1}$, the equations [17]

$$
\frac{\mathrm{d}}{\mathrm{d} \tau} \rho_{k}^{(n)}=\sigma\left[(k+p) \rho_{k+p}^{(n)}-(k-p) \rho_{k-p}^{(n)}\right]+2 i \gamma k \rho_{k}^{(n)}
$$

where $\gamma \equiv \delta / \varepsilon, \sigma \equiv(-1)^{p}$, and $\tau=\frac{1}{2} \varepsilon \omega_{1} t$ is the 'slow time'. The exact solutions to equation (2.4) differ from the approximate form (2.5) by the terms proportional to the higher harmonics $\exp \left[ \pm i r \omega_{k} t\right], r=2,3, \ldots$ However, the magnitudes of the corrections are of the order of $\varepsilon$ (or less), so they can be neglected, at least under the condition $t \ll t_{2} \sim\left(\omega_{1} \varepsilon^{2}\right)^{-1}$. For the values $\omega_{1} \sim 10^{10} \mathrm{~s}^{-1}$ and $\varepsilon \sim 10^{-8}$ corresponding to the possible experimental realisations [5] the limiting time $t_{2}$ is of the order of weeks.

Due to the initial conditions $\rho_{k}^{(n)}(0)=\delta_{k n}$ the solutions to (2.6) satisfy the relation $\rho_{j+m p}^{(k+n p)} \equiv 0$ if $j \neq k$. The non-zero coefficients $\rho_{m}^{(n)}$ read [17]

$$
\begin{aligned}
\rho_{j+m p}^{(j+n p)}(\tau)= & \frac{\Gamma(1+n+j / p)(\sigma \kappa)^{n-m} \lambda^{m+n+2 j / p}}{\Gamma(1+m+j / p) \Gamma(1+n-m)} \\
& \times F\left(n+j / p,-m-j / p ; 1+n-m ; \kappa^{2}\right)
\end{aligned}
$$

where $F(a, b ; c ; z)$ is the Gauss hypergeometric function,

$$
\kappa=\frac{\sinh (a p \tau)}{\sqrt{a^{2}+\sinh ^{2}(a p \tau)}}, \quad a=\sqrt{1-\gamma^{2}}, \quad \lambda=\sqrt{1-\gamma^{2} \kappa^{2}}+i \gamma \kappa .
$$


The functions (2.7) are exact solutions to the set of equations (2.6) relating the coefficients with different lower indices. Besides, these functions satisfy another set of equations, which can be treated as recurrence relations with respect to the upper indices [17]

$$
\begin{array}{ll}
\frac{d}{d \tau} \rho_{m}^{(n)}=n\left\{\sigma\left[\rho_{m}^{(n-p)}-\rho_{m}^{(n+p)}\right]+2 i \gamma \rho_{m}^{(n)}\right\}, & n \geq p, \quad \rho_{m}^{(0)} \equiv 0 \\
\frac{d}{d \tau} \rho_{m}^{(n)}=n\left\{\sigma\left[\rho_{-m}^{(p-n) *}-\rho_{m}^{(p+n)}\right]+2 i \gamma \rho_{m}^{(n)}\right\}, & n=1,2, \ldots, p-1
\end{array}
$$

The consequences of equations (2.6), (2.9) and (2.10) are the identities

$$
\begin{aligned}
& \sum_{m=-\infty}^{\infty} m \rho_{m}^{(n) *} \rho_{m}^{(k)}=n \delta_{n k}, \quad n, k=1,2, \ldots \\
& \sum_{n=1}^{\infty} \frac{m}{n}\left[\rho_{m}^{(n) *} \rho_{j}^{(n)}-\rho_{-m}^{(n) *} \rho_{-j}^{(n)}\right]=\delta_{m j}, \quad m, j=1,2, \ldots \\
& \sum_{n=1}^{\infty} \frac{1}{n}\left[\rho_{m}^{(n) *} \rho_{-j}^{(n)}-\rho_{j}^{(n) *} \rho_{-m}^{(n)}\right]=0, \quad m, j=1,2, \ldots
\end{aligned}
$$

The formulas given above hold for any value of the detuning parameter $\gamma$. For $\gamma>1$ one should replace the functions $\sinh (a x) / a$ and $\cosh (a x)$ by their trigonometrical counterparts $\sin (\tilde{a} x) / \tilde{a}$ and $\cos (\tilde{a} x)$, where $\tilde{a}=\sqrt{\gamma^{2}-1}$.

\section{Squeezing the initial vacuum}

We suppose that after some interval of time $T$ the wall comes back to its initial position $L_{0}$. For $t \geq T$, the field operator assumes the form

$$
\hat{A}(x, t)=\sum_{n=1}^{\infty} \frac{2}{\sqrt{n}} \sin \left(\pi n x / L_{0}\right)\left[\hat{a}_{n} e^{-i \omega_{n}(t+\delta T)}+\text { h.c. }\right]
$$

where operators $\hat{a}_{m}$ are related to the initial operators $\hat{b}_{n}$ and $\hat{b}_{n}^{\dagger}$ by means of the Bogoliubov transformation $\left(\tau_{T} \equiv \frac{1}{2} \varepsilon \omega_{1} T\right)$

$$
\hat{a}_{m}=\sum_{n=1}^{\infty} \sqrt{\frac{m}{n}}\left[\hat{b}_{n} \rho_{m}^{(n)}\left(\tau_{T}\right)-\hat{b}_{n}^{\dagger} \rho_{-m}^{(n) *}\left(\tau_{T}\right)\right], \quad m=1,2, \ldots
$$

The commutation relations $\left[\hat{a}_{n}, \hat{a}_{k}^{\dagger}\right]=\delta_{n k}$ hold due to the identities (2.11)-(2.13) which are nothing but the unitarity conditions of the transformation (3.2). These commutation relations together with the expression for the energy of the field

$$
\hat{H} \equiv \frac{1}{8 \pi} \int_{0}^{L_{0}} \mathrm{~d} x\left[\left(\frac{\partial \hat{A}}{\partial t}\right)^{2}+\left(\frac{\partial \hat{A}}{\partial x}\right)^{2}\right]=\sum_{n=1}^{\infty} \omega_{n}\left(\hat{a}_{n}^{\dagger} \hat{a}_{n}+\frac{1}{2}\right)
$$

convince us that namely $\hat{a}_{n}$ and $\hat{a}_{n}^{\dagger}$ are true photon annihilation and creation operators at $t \geq T$ (like the operators $\hat{b}_{n}$ and $\hat{b}_{n}^{\dagger}$ were 'physical' ones at $t<0$ ).

Now we introduce the Hermitian quadrature component operators

$$
\hat{q}_{m}=\left(\hat{a}_{m}+\hat{a}_{m}^{\dagger}\right) / \sqrt{2}, \quad \hat{p}_{m}=\left(\hat{a}_{m}-\hat{a}_{m}^{\dagger}\right) /(i \sqrt{2})
$$


and calculate their variances $U_{m}=\left\langle\hat{q}_{m}^{2}\right\rangle-\left\langle\hat{q}_{m}\right\rangle^{2}, V_{m}=\left\langle\hat{p}_{m}^{2}\right\rangle-\left\langle\hat{p}_{m}\right\rangle^{2}$ and the covariance $Y_{m}=\frac{1}{2}\left\langle\hat{p}_{m} \hat{q}_{m}+\hat{q}_{m} \hat{p}_{m}\right\rangle-\left\langle\hat{p}_{m}\right\rangle\left\langle\hat{q}_{m}\right\rangle$ in the vacuum state with respect to the initial operators $\hat{b}_{n}: \hat{b}_{n}|0\rangle=0$ (remember that we use the Heisenberg picture). Using (3.2) we obtain (assuming hereafter $\omega_{1}=1$ )

$$
\begin{aligned}
U_{m} & =\frac{m}{2} \sum_{n=1}^{\infty} \frac{1}{n}\left|\rho_{m}^{(n)}-\rho_{-m}^{(n)}\right|^{2}, \quad V_{m}=\frac{m}{2} \sum_{n=1}^{\infty} \frac{1}{n}\left|\rho_{m}^{(n)}+\rho_{-m}^{(n)}\right|^{2} \\
Y_{m} & =\sum_{n=1}^{\infty} \frac{m}{n} \operatorname{Im}\left[\rho_{m}^{(n) *} \rho_{-m}^{(n)}\right]
\end{aligned}
$$

where the coefficients $\rho_{ \pm m}^{(n)}$ should be taken at the moment $T$, thus their argument is $\tau_{T}$. Strictly speaking, the expressions (3.4)-(3.5) have physical meanings at those moments of time $T$ when the wall returns to its initial position, i.e. for $T=N \pi /[p(1+\delta)]$ with an integer $N$. Consequently, the argument $\tau_{T}$ of the coefficients $\rho_{ \pm m}^{(n)}$ in (3.4)-(3.5) assumes discrete values $\tau^{(N)}=N \varepsilon \pi /[2 p(1+\delta)]$. One should remember, however, that something interesting in our problem happens for the values $\tau \sim 1$ (or larger). Then $N \sim \varepsilon^{-1} \gg 1$, and the minimal increment $\Delta \tau \sim \varepsilon$ is so small that $\tau_{T}$ can be considered as a continuous variable (under the realistic conditions, $\varepsilon \leq 10^{-8}$ [5]). For this reason, we omit hereafter the subscript $T$, writing simply $\tau$ instead of $\tau_{T}$ or $\tau^{(N)}$.

Differentiating the right-hand sides of equations (3.4) and (3.5) with respect to the 'slow time' $\tau$, one can remove the fraction $1 / n$ with the aid of the recurrence relations (2.9) and (2.10). After that, changing if necessary the summation index $n$ to $n \pm p$, one can verify that almost all terms in the right-hand sides are cancelled, and the infinite series are reduced to the finite sums:

$$
\begin{aligned}
& \left.\begin{array}{l}
\mathrm{d} U_{m} / \mathrm{d} \tau \\
\mathrm{d} V_{m} / \mathrm{d} \tau
\end{array}\right\}=\sigma m \sum_{n=1}^{p-1} \operatorname{Re}\left(\left[\rho_{m}^{(p-n)} \mp \rho_{-m}^{(p-n)}\right]\left[\rho_{-m}^{(n)} \mp \rho_{m}^{(n)}\right]\right) \\
& \mathrm{d} Y_{m} / \mathrm{d} \tau=\sigma m \sum_{n=1}^{p-1} \operatorname{Im}\left(\rho_{m}^{(n) *} \rho_{m}^{(p-n) *}+\rho_{-m}^{(n)} \rho_{-m}^{(p-n)}\right)
\end{aligned}
$$

Now one should take into account the structure of the coefficients $\rho_{m}^{(n)}$ (2.7): they are different from zero provided the difference between the upper index $n$ and the lower one $m$ is some multiple of the number $p$. If $m=j+p k$ with $j=1, \ldots, p-1$ and $k=0,1,2, \ldots$, then only the terms with $n=j$ or $n=p-j$ survive in the sums above. Depending on whether $j=p / 2$ or $j \neq p / 2$, we obtain two different sets of explicit expressions for the derivatives of the (co)variances.

1) If $m=j+p k$ but $j \neq p / 2$ (in particular, for all odd values of $p$ ), then

$$
\frac{\mathrm{d} U_{m}}{\mathrm{~d} \tau}=\frac{\mathrm{d} V_{m}}{\mathrm{~d} \tau}=2 \sigma m \operatorname{Re}\left(\rho_{m}^{(j)} \rho_{-m}^{(p-j)}\right), \quad \frac{\mathrm{d} Y_{m}}{\mathrm{~d} \tau}=0 .
$$

In this case $Y_{m} \equiv 0$ and $U_{m}=V_{m}=\mathcal{N}_{m}+1 / 2$, where $\mathcal{N}_{m}$ is the mean number of photons created from vacuum in the $m$ th mode calculated in [17], so there is no squeezing. For $\gamma \leq 1$ the quadrature variances monotonously increase in time, with an asymptotical linear dependence. If $\gamma>1$, the variances oscillate in time with amplitudes inversly proportional to $\gamma^{2}-1$, being always not less than $1 / 2$. 
2) Some squeezing can be achieved only in the 'principal' modes with the numbers $\mu=p(k+1 / 2), k=0,1,2, \ldots$ :

$$
\begin{aligned}
& \frac{\mathrm{d} U_{\mu}}{\mathrm{d} \tau}=-\mu \operatorname{Re}\left(\left[\rho_{\mu}^{(p / 2)}-\rho_{-\mu}^{(p / 2)}\right]^{2}\right), \quad \frac{\mathrm{d} V_{\mu}}{\mathrm{d} \tau}=\mu \operatorname{Re}\left(\left[\rho_{\mu}^{(p / 2)}+\rho_{-\mu}^{(p / 2)}\right]^{2}\right) \\
& \mathrm{d} Y_{\mu} / \mathrm{d} \tau=\mu \operatorname{Im}\left(\left[\rho_{\mu}^{(p / 2) *}\right]^{2}+\left[\rho_{-\mu}^{(p / 2)}\right]^{2}\right)
\end{aligned}
$$

(in particular, it is necessary that $p$ be an even number). In the strict resonance case $(\gamma=0)$ all the coefficients $\rho_{\mu}^{(p / 2)}$ are real, so $Y_{\mu}=0$ and $d U_{\mu} / d \tau \leq 0$ in the whole interval $0 \leq \tau<\infty$, resulting in the inequality $U_{\mu}(\tau)<1 / 2$.

Note that the coefficients $\rho_{p m+p / 2}^{(p n+p / 2)}$ depend on the parameter $p$ only through the dependence of the the variable $\kappa$ on the product $p \tau$ : see equation (2.8) and the explicit form of these coefficients in Appendix A. Thus it is sufficient to consider the most important special case of the parametric resonance at the double fundamental frequency $2 \omega_{1}$ (i.e. $p=2$ ), since the formulae for $p>2$ can be obtained by a simple rescaling of the 'slow time' (for the 'principal' modes). In this case, only the odd modes can be excited from the vacuum, and they do exhibit some squeezing.

Using equations (3.7) and (3.8) one can immediately find the Taylor expansions of the (co)variances at $\tau \rightarrow 0$ (assuming $(-1) ! ! \equiv 1$ ):

$$
\begin{aligned}
& \left.\begin{array}{l}
U_{2 m+1} \\
V_{2 m+1}
\end{array}\right\}=\frac{1}{2} \mp \tau^{2 m+1}\left[\frac{(2 m-1) ! !}{m !}\right]^{2}\left[1 \mp \frac{2 m+1}{(m+1)^{2}} \tau+\mathcal{O}\left(\tau^{2}\right)\right] \\
& Y_{2 m+1}=-2 \gamma(2 m+1) \tau^{2(m+1)}\left[\frac{(2 m-1) ! !}{m !}\right]^{2}+\cdots
\end{aligned}
$$

We see that the $U$-variances are always less than $1 / 2$ at the initial stage, but the degree of their squeezing rapidly decreases with increase of the number $m$. Note that the dependence on the detuning parameter $\gamma$ in the short-time limit appears only in terms of the order of $\tau^{2 m+3}$ (and higher).

In the opposite limit $\tau \rightarrow \infty$ (or $\kappa \rightarrow 1$ ), using equations (3.7), (3.8) and the asymptotics of the Bogoliubov coefficients (A.4) we obtain constant time derivatives

$$
\begin{aligned}
\mathrm{d} U_{2 m+1} /\left.\mathrm{d} \tau\right|_{\tau \rightarrow \infty} & =\frac{16 a}{\pi^{2}(2 m+1)} \sin ^{2}\left[\left(m+\frac{1}{2}\right) \phi\right] \\
\mathrm{d} V_{2 m+1} /\left.\mathrm{d} \tau\right|_{\tau \rightarrow \infty} & =\frac{16 a}{\pi^{2}(2 m+1)} \cos ^{2}\left[\left(m+\frac{1}{2}\right) \phi\right] \\
\mathrm{d} Y_{2 m+1} /\left.\mathrm{d} \tau\right|_{\tau \rightarrow \infty} & =-\frac{8 a}{\pi^{2}(2 m+1)} \sin [(2 m+1) \phi]
\end{aligned}
$$

where $\phi \equiv \arcsin \gamma$. Consequently, all the (co)variances increase with time linearly, giving the constant photon generation rate in the 'principal' (odd) modes

$$
\mathrm{d} \mathcal{N}_{2 m+1} /\left.\mathrm{d} \tau\right|_{\tau \rightarrow \infty}=\frac{8 a}{\pi^{2}(2 m+1)}
$$

in agreement with [17]. Equation (3.14) results in a simple estimation of the mean photon number in the $\mu$ th mode at $\tau>1: \mathcal{N}_{\mu}(\tau) \approx a \tau / \mu$. 
Since the covariance $Y_{\mu}$ is different from zero if $\gamma \neq 0$, the initial vacuum state of the field is transformed to the correlated quantum state [32, 33]. One should remember, however, that the values of $U_{\mu}, V_{\mu}$ and $Y_{\mu}$ yield the (co)variances of the field quadratures only at the moment $t=T$ (when the wall stopped to oscillate). At the subsequent moments of time the quadrature variances exhibit fast oscillations with twice the frequency of the mode. For example (omitting the mode index),

$$
\sigma_{q}\left(t^{\prime}\right)=U \cos ^{2}\left(\omega t^{\prime}\right)+V \sin ^{2}\left(\omega t^{\prime}\right)+Y \sin \left(2 \omega t^{\prime}\right), \quad t^{\prime}=t-T .
$$

Therefore the physical meanings have not the values $U_{\mu}, V_{\mu}$ and $Y_{\mu}$ themselves, but rather the minimal $\sigma_{\min } \equiv u_{\mu}$ and maximal $\sigma_{\max } \equiv v_{\mu}$ values of the quadrature variances during the period of fast oscillations [34]

$$
\left.\begin{array}{l}
u_{\mu} \\
v_{\mu}
\end{array}\right\}=\frac{1}{2}\left(U_{\mu}+V_{\mu} \mp \sqrt{\left(U_{\mu}-V_{\mu}\right)^{2}+4 Y_{\mu}^{2}}\right) .
$$

Only in the special case of the strict resonane $(\gamma=0)$ we have $u_{\mu}=U_{\mu}$ and $v_{\mu}=V_{\mu}$.

The equations (3.7) and (3.8) can be integrated for any values of $\mu$ and $\gamma$ in terms of the complete elliptic integrals $\mathbf{K}(\kappa)$ and $\mathbf{E}(\kappa)$ : see Appendix B for the technical details. In particular, for the fundamental mode we find

$$
\begin{aligned}
& U_{1}=\frac{2}{\pi^{2} \kappa}\left[\tilde{\kappa}^{2}(\beta-\kappa) \mathbf{K}^{2}(\kappa)-2(\beta-\kappa) \mathbf{K}(\kappa) \mathbf{E}(\kappa)+\beta \mathbf{E}^{2}(\kappa)\right], \\
& V_{1}=\frac{2}{\pi^{2} \kappa}\left[2(\beta+\kappa) \mathbf{K}(\kappa) \mathbf{E}(\kappa)-\tilde{\kappa}^{2}(\beta+\kappa) \mathbf{K}^{2}(\kappa)-\beta \mathbf{E}^{2}(\kappa)\right], \\
& Y_{1}=\frac{2 \gamma}{\pi^{2}}\left[\tilde{\kappa}^{2} \mathbf{K}^{2}(\kappa)-2 \mathbf{K}(\kappa) \mathbf{E}(\kappa)+\mathbf{E}^{2}(\kappa)\right]
\end{aligned}
$$

where $\beta=\operatorname{Re} \lambda=\sqrt{1-\gamma^{2} \kappa^{2}}$ and $\tilde{\kappa} \equiv \sqrt{1-\kappa^{2}}$. Then equation (3.15) yields the minimal and maximal invariant variances

$$
\begin{aligned}
& u_{1}=\frac{2}{\pi^{2} \kappa}\left[\tilde{\kappa}^{2}(1-\kappa) \mathbf{K}^{2}(\kappa)-2(1-\kappa) \mathbf{K}(\kappa) \mathbf{E}(\kappa)+\mathbf{E}^{2}(\kappa)\right] \\
& v_{1}=\frac{2}{\pi^{2} \kappa}\left[2(1+\kappa) \mathbf{K}(\kappa) \mathbf{E}(\kappa)-\tilde{\kappa}^{2}(1+\kappa) \mathbf{K}^{2}(\kappa)-\mathbf{E}^{2}(\kappa)\right]
\end{aligned}
$$

which depend on the detuning parameter $\gamma$ only implicitly, through the dependence on $\gamma$ of the function $\kappa(\tau)$ (2.8). In the short time limit $\tau \ll 1$ (then $\kappa \approx 2 \tau$ ) we obtain, using the Taylor expansions of the complete elliptic integrals, $u_{1}=\frac{1}{2}-\tau+\tau^{2}+\cdots$ and $v_{1}=\frac{1}{2}+\tau+\tau^{2}+\cdots$ in accordance with [26]. More precisely,

$$
\left.\begin{array}{l}
u_{1} \\
v_{1}
\end{array}\right\}=\frac{1}{2}\left(1 \mp \kappa+\frac{1}{2} \kappa^{2} \mp \frac{1}{4} \kappa^{3}+\frac{7}{32} \kappa^{4}+\cdots\right)
$$

The minimal variance $u_{1}$ monotonously decreases from the value $1 / 2$ at $t=0$ to the constant asymptotical value $2 / \pi^{2}$ at $\tau \gg 1$, confirming qualitatively the evaluations of [28, 29] and giving almost 50\% squeezing in the initial vacuum state. The variance of the conjugate quadrature monotonously increases, and for $\tau \gg 1$ it becomes practically linear function of time: $v_{1}(\tau \gg 1) \approx 16 \tau / \pi^{2}$. The asymptotical minimal value 
$u_{1}(\tau=\infty)$ does not depend on $\gamma$ provided $\gamma \leq 1$ (only the rate of reaching this asymptotical value decreases with $\gamma$ as $\sqrt{1-\gamma^{2}}$ ). In the strongly detuned case, $\gamma>1$, the minimal variance oscillates as a function of $\tau$ (being always greater than $2 / \pi^{2}$ ), since in this case the function $\kappa(\tau)$ oscillates between $-\gamma^{-1}$ and $\gamma^{-1}$.

The minimal variance does not go to zero when $\tau \rightarrow \infty$ due to the strong intermode interaction, which results in a high degree of quantum mixing for each mode. Since the state originating from the initial vacuum state belongs to the class of Gaussian states (see section 5), the quantum 'purity' $\chi_{m} \equiv \operatorname{Tr} \hat{\rho}_{m}^{2}$ of the $m$ th field mode (described by means of the density matrix $\hat{\rho}_{m}$ ) can be expressed in terms of the (co)variances as [35] $\chi_{m}=\left[4\left(U_{m} V_{m}-Y_{m}^{2}\right)\right]^{-1 / 2}$. Using equations (3.11)-(3.13) one can check that $\chi \sim \tau^{-1 / 2} \rightarrow 0$ for $\tau \gg 1$ (see Appendix C). For instance, for $m=1$ we have (writing simply $\mathbf{K}$ and $\mathbf{E}$ instead of $\mathbf{K}(\kappa)$ and $\mathbf{E}(\kappa))$

$$
\chi_{1}=\frac{\pi^{2}}{4} \kappa\left[4 \mathbf{K E}^{3}+4 \tilde{\kappa}^{4} \mathbf{K}^{3} \mathbf{E}-6 \tilde{\kappa}^{2} \mathbf{K}^{2} \mathbf{E}^{2}-\mathbf{E}^{4}-\tilde{\kappa}^{6} \mathbf{K}^{4}\right]^{-1 / 2}
$$

The initial dependence on $\kappa$ is rather weak: $\chi(\kappa \ll 1)=1-\frac{3}{32} \kappa^{4}+\cdots$. But when $\kappa \rightarrow 1, \chi$ rapidly goes to zero: $\chi(\tilde{\kappa} \ll 1) \approx\left(8 / \pi^{2}\right)[\ln (4 / \tilde{\kappa})]^{-1 / 2}$, with $\mathrm{d} \chi / \mathrm{d} \kappa \rightarrow-\infty$.

It is worth to mention that in the resonance case there is no effective interaction between different modes of a nondegenerate (three-dimensional) cavity possessing nonequidistant eigenmode spectrum. In such a cavity the minimal variance asymptotically goes to zero for $\tau \rightarrow \infty$ [5, 15].

For the sake of completeness we bring the formulae (found in [17]) for the mean photon number in the fundamental mode and the total energy of photons created in all the modes (for the initial vacuum state):

$$
\begin{aligned}
& \mathcal{N}_{1}(\kappa)=\frac{2}{\pi^{2}} \mathbf{K}(\kappa)\left[2 \mathbf{E}(\kappa)-\tilde{\kappa}^{2} \mathbf{K}(\kappa)\right]-\frac{1}{2} \\
& \mathcal{E}_{\text {tot }}(\tau)=\frac{p^{2}-1}{12 a^{2}} \sinh ^{2}(p a \tau) .
\end{aligned}
$$

In [17] it was derived also the formula for the second derivative of the total mean number of photons created from vacuum in all the modes

$$
\frac{\mathrm{d}^{2} \mathcal{N}_{t o t}}{\mathrm{~d} \tau^{2}}=\frac{8}{\pi^{2} \kappa^{2}}\left[\widetilde{\kappa}^{4} \mathbf{K}^{2}-2 \widetilde{\kappa}^{2} \mathbf{K E}+\left(1+\kappa^{2}-2 \gamma^{2} \kappa^{4}\right) \mathbf{E}^{2}\right]
$$

Now we are able to integrate this equation. Taking into account the condition [17] $\mathrm{d} \mathcal{N} / \mathrm{d} \tau=0$ at $\tau=0$ we obtain very simple expression

$$
\mathcal{N}_{\text {tot }}=\frac{2}{\pi^{2}} \mathbf{K}(\kappa)[\mathbf{K}(\kappa)-\mathbf{E}(\kappa)]
$$

The expressions for the variances in the modes with numbers $\mu \geq 3$ are rather involved; their general structure is discussed in Appendix B. Here we give only one explicit example - the variance $U_{3}$ for $\gamma=0$ :

$$
\begin{aligned}
U_{3}= & \frac{2}{9 \pi^{2} \kappa^{3}}\left[\tilde{\kappa}^{2}(1-\kappa)\left(4+10 \kappa+9 \kappa^{2}\right) \mathbf{K}^{2}(\kappa)\right. \\
& +(1-\kappa)\left(4 \kappa^{3}-14 \kappa^{2}-20 \kappa-8\right) \mathbf{K}(\kappa) \mathbf{E}(\kappa) \\
& \left.+\left(4 \kappa^{4}+6 \kappa^{3}-\kappa^{2}+6 \kappa+4\right) \mathbf{E}^{2}(\kappa)\right]
\end{aligned}
$$


The Taylor expansion of the right-hand side of (3.23) coincides with the expansion (3.9). The asymptotical value at $\tau \rightarrow \infty$ equals $U_{3}(\kappa=1)=38 /\left(9 \pi^{2}\right) \approx 0.43$. We see that the squeezing rapidly disappears with increase of the mode number $\mu$.

The variance $V_{3}$ can be obtained from (3.23) by means of a simple substitution $\kappa \rightarrow-\kappa$. Therefore the mean number of photons in the third mode is given by

$$
\mathcal{N}_{3}=\frac{2}{3 \pi^{2} \kappa^{2}}\left[\left(3 \kappa^{2}-2\right) \mathbf{K}\left(2 \mathbf{E}-\tilde{\kappa}^{2} \mathbf{K}\right)+2\left(1+\kappa^{2}\right) \mathbf{E}^{2}\right]-\frac{1}{2} .
$$

It is remarkable that despite all three (co)variances $U_{\mu}, V_{\mu}$ and $Y_{\mu}$ linearly increase with time at $\tau \gg 1$ in the generic case $\gamma \neq 0$, the minimal variance $u_{\mu}$ tends to a constant value at $\tau \rightarrow \infty$ : see Appendix $\mathrm{C}$.

The results of this section confirm completely the earlier conclusions 26] concerning the behaviour of the quadrature variances in the short-time limit for $p=2$, as well as the results of approximate numerical calculations performed in [27] (in the same limit) for $p=2$ and $p=3$. In the long-time limit we see a qualitative agreement with earlier approximate asymptotical formulae of [28, 29] related to the behaviour of the squeezed quadrature component variance (for $\gamma=0$ ), namely, that the squeezing effect is strongest for the lowest mode and that it decreases with increase of the mode number. However, there are differences in the numerical values of the squeezed variances. This can be explained as follows. In 28, 29 only the leading terms of the Bogoliubov coefficients (analogs of the coefficients $\rho_{m}^{(n)}$ ) were found. But the coefficients $\rho_{m}^{(n)}(\kappa)$ and $\rho_{-m}^{(n)}(\kappa)$ become the same in the limit $\kappa=1$ (if $\lambda=1$ ) and they do not depend on the upper index $n$ : see Appendix A. For this reason, although the leading terms of the asymptotic expansions enable to calculate correctly the number of photons and the unsqueezed variances like $V_{2 m+1}$, these terms are canceled in the expressions for the squeezed variances like $U_{2 m+1}$ (if $\gamma=0$ ). It was found in 28, 29] that the difference $\Delta_{m} \equiv \frac{1}{2}-U_{2 m+1}(\tau \rightarrow \infty)$ decreases as $1 /(2 m+1)$ for $m \gg 1$. The numerical integration of equation (3.7) yields for the product $(2 m+1) \Delta_{m}$ the values close to 0.2 for $1 \leq m \leq 5$ (according to (3.23), 3 $\Delta_{1}=0.2166 \ldots$ ). It seems probable that the limit value of this product at $m \rightarrow \infty$ equals $2 / \pi^{2}=0.2026 \ldots$, but we did not succeed to prove this conjecture analytically.

\section{Influence of initial conditions}

For an arbitrary initial state of the field one can write $U_{m}=U_{m}^{(v a c)}+\Delta U_{m}$, where $U_{m}^{(v a c)}$ is given by equation (3.4); similar expressions can be written for $V_{m}$ and $Y_{m}$. The corrections due to the nonvacuum initial states are given by

$$
\begin{gathered}
\left.\begin{array}{c}
\Delta U_{m} \\
\Delta V_{m}
\end{array}\right\}=\operatorname{Re} \sum_{n, j} \frac{m}{\sqrt{n j}}\left(\left[\rho_{m}^{(n)} \mp \rho_{-m}^{(n)}\right]^{*}\left[\rho_{m}^{(j)} \mp \rho_{-m}^{(j)}\right]\left[\left\langle\hat{b}_{n}^{\dagger} \hat{b}_{j}\right\rangle-\left\langle\hat{b}_{n}^{\dagger}\right\rangle\left\langle\hat{b}_{j}\right\rangle\right]\right. \\
\left. \pm\left[\rho_{m}^{(n)} \mp \rho_{-m}^{(n)}\right]\left[\rho_{m}^{(j)} \mp \rho_{-m}^{(j)}\right]\left[\left\langle\hat{b}_{n} \hat{b}_{j}\right\rangle-\left\langle\hat{b}_{n}\right\rangle\left\langle\hat{b}_{j}\right\rangle\right]\right) \\
\Delta Y_{m}=\operatorname{Im} \sum_{n, j} \frac{m}{\sqrt{n j}}\left(\left[\rho_{m}^{(n) *} \rho_{-m}^{(j)}-\rho_{m}^{(j)} \rho_{-m}^{(n) *}\right]\left[\left\langle\hat{b}_{n}^{\dagger} \hat{b}_{j}\right\rangle-\left\langle\hat{b}_{n}^{\dagger}\right\rangle\left\langle\hat{b}_{j}\right\rangle\right]\right.
\end{gathered}
$$




$$
\left.+\left[\rho_{m}^{(n)} \rho_{m}^{(j)}-\rho_{-m}^{(n)} \rho_{-m}^{(j)}\right]\left[\left\langle\hat{b}_{n} \hat{b}_{j}\right\rangle-\left\langle\hat{b}_{n}\right\rangle\left\langle\hat{b}_{j}\right\rangle\right]\right)
$$

where the average values like $\left\langle\hat{b}_{n}^{\dagger} \hat{b}_{j}\right\rangle$ are calculated in the initial state. All the corrections disappear in the case of the initial coherent state, $\hat{b}_{n}|\alpha\rangle=\alpha_{n}|\alpha\rangle$. If the initial density matrix is diagonal in the Fock basis (as happens e.g. for the Fock or thermal states) then $\left\langle\hat{b}_{n}^{\dagger} \hat{b}_{j}\right\rangle=\nu_{n} \delta_{n j}\left(\nu_{n} \geq 0\right)$, all other average values in (4.1) and (4.2) being equal to zero. In this case the double sums are reduced to the single ones:

$$
\begin{aligned}
& \Delta U_{m}=m \sum_{n} \frac{\nu_{n}}{n}\left|\rho_{m}^{(n)}-\rho_{-m}^{(n)}\right|^{2}, \quad \Delta V_{m}=m \sum_{n} \frac{\nu_{n}}{n}\left|\rho_{m}^{(n)}+\rho_{-m}^{(n)}\right|^{2} \\
& \Delta Y_{m}=2 m \sum_{n} \frac{\nu_{n}}{n} \operatorname{Im}\left[\rho_{m}^{(n) *} \rho_{-m}^{(n)}\right] .
\end{aligned}
$$

We see that the initial fluctuations always increase both the variances $U_{m}$ and $V_{m}$ (for the diagonal density matrix). However, asymptotically at $\tau \rightarrow \infty$ the corrections are bounded for the physical initial states having finite total numbers of photons, because the coefficients $\left|\rho_{m}^{(n)} \pm \rho_{-m}^{(n)}\right|^{2}$ and $\operatorname{Im}\left[\rho_{m}^{(n) *} \rho_{-m}^{(n)}\right]$ do not depend on the summation index $n$ in this limit: see equations (A.6) and (A.7). Thus we have in the 'principal' $\mu$-modes (for $p=2 ; \phi \equiv \arcsin \gamma$ )

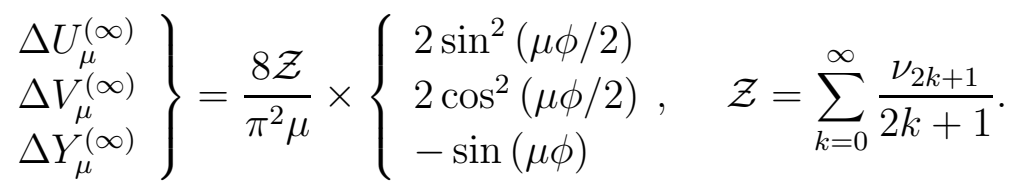

These expressions are very similar to (3.11)-(3.13). Their consequence is the important result that in the limit $\tau \rightarrow \infty$ the minimal variance $u_{\mu}$ does not depend on the initial state of the field inside the cavity, provided the initial density matrix was diagonal in the Fock basis. The proof is given in Appendix Q. The correction to the mean number of photons tends to the limit $\Delta \mathcal{N}_{\mu}^{(\infty)}=8 \mathcal{Z} /\left(\pi^{2} \mu\right)$.

\section{Photon distribution}

Now let us turn to the photon distribution function (PDF) $f(\mathbf{n}) \equiv\left\langle\mathbf{n}\left|\hat{\rho}_{m}(t)\right| \mathbf{n}\right\rangle$, where $|\mathbf{n}\rangle$ is the multimode Fock state, $\mathbf{n} \equiv\left(n_{1}, n_{2}, \ldots\right)$, and $\hat{\rho}_{m}(t)$ is the time-dependent density matrix of the $m$ th field mode in the Schrödinger picture. At first glance, there is a problem, since all the calculations in the preceding sections were performed in the framework of the Heisenberg picture. Fortunately, this problem can be easily resolved for a special class of Gaussian initial states (i.e. the states whose density matrices, or wave functions, or Wigner functions, are described by some Gaussian exponentials). This class includes coherent, squeezed and thermal states; in particular, it includes the vacuum state which we are interested in here.

The solution is based on two key points. The first one is the statement [9, 31] that the field evolution in a cavity with moving boundaries can be described not only in the Heisenberg picture, but, equivalently, in the framework of the Schrödinger picture, with a quadratic multidimensional time-dependent Hamiltonian. The second key point 
is the fact 36, 37 that the evolution governed by quadratic Hamiltonians transforms any Gaussian state to another Gaussian state.

It remains to take into account that the photon distribution function of any Gaussian state is determined completely by the average values of quadratures and by their variances 38, 39, which obviously do not depend on the quantum mechanical representation. The explicit formulae in the generic case are rather involved, so we give them in Appendix D. Here we confine ourselves to the most simple case of the vacuum initial state, when all the average values of quadratures are equal to zero. In this case the generating function $(\overline{D .1})$ is reduced to $[\mathcal{G}(z)]^{-1 / 2}$, i.e. it has the same structure as the known generating function of the Legendre polynomials $P_{n}(x)$. After some algebra we obtain the following expression for the photon distribution in the $m$ th field mode:

$$
f_{m}(n)=\frac{2\left[\left(2 u_{m}-1\right)\left(2 v_{m}-1\right)\right]^{n / 2}}{\left[\left(2 u_{m}+1\right)\left(2 v_{m}+1\right)\right]^{(n+1) / 2}} P_{n}\left(\frac{4 u_{m} v_{m}-1}{\sqrt{\left(4 u_{m}^{2}-1\right)\left(4 v_{m}^{2}-1\right)}}\right)
$$

It depends only on the invariant minimal and maximal variances $u_{m}$ and $v_{m}$. Note that the argument of the polynomial in (5.1) is always outside the 'traditional' interval $(-1,1)$ (in particular, this argument is pure imaginary if $2 u_{m}<1$ ), being exactly equal to 1 for the 'non-principal' modes with $u_{m}=v_{m}=\mathcal{N}_{m}+\frac{1}{2}$, when formula (5.1) transforms to the time-dependent Planck's distribution

$$
f_{m}(n ; \tau)=\frac{\mathcal{N}_{m}^{n}(\tau)}{\left[\mathcal{N}_{m}(\tau)+1\right]^{n+1}}
$$

Only for the 'principal' $\mu$-modes the spectrum of photons is different from Planck's one due to the squeezing effect. The first and second derivatives of the generating function (D.1) at $z=1$ yield the first two moments of the photon distribution (hereafter we suppress subscript $m$ )

$$
\bar{n}=\frac{1}{2}(u+v-1), \quad \sigma_{n} \equiv \overline{n^{2}}-(\bar{n})^{2}=\frac{1}{4}\left(2 u^{2}+2 v^{2}-1\right)
$$

which result in the Mandel parameter

$$
Q \equiv \sigma_{n} / \bar{n}-1=\frac{u^{2}+v^{2}-u-v+1 / 2}{u+v-1}
$$

This parameter appears positive for all values of $\tau$, so the photon statistics is superPoissonian, with strong bunching of photons (the pair creation of photons in the NSCE was discussed in [3, 4, 7, 13]). In particular,

$$
Q_{2 m+1}(\tau \rightarrow 0) \approx[(m+1)(2 m-1) ! ! / m !]^{2} \tau^{2 m} /(2 m+1), \quad Q_{1}(0)=1,
$$

whereas $Q_{m} \approx V_{m}(\tau) \gg 1$ for $\tau \gg 1$ (if $\gamma \ll 1$ ).

The analytical expressions obtained in the paper are illustrated in two figures. In figure 11 we show the dependences on the universal variable $\kappa(2.8)$ of the minimal and maximal variances $u_{1}$ (3.16) and $v_{1}$ (3.17) together with the purity factor $\chi_{1}$ (3.18), the mean photon number $\mathcal{N}_{1}(3.19)$ and the $Q$-factor (5.3) for the fundamental mode $\mu=1$. An example of the photon distribution in the principal mode $\mu=1$ for $\gamma=0$ is given in figure 2, where the 'cavity' distribution is compared with Planck's one corresponding 
to the same mean photon number. We see no oscillations typical for the pure squeezed states [40-42] (excluding a small 'splash' at $n=2$ ), because the field appears in a mixed quantum state (the influence of quantum mixing on the oscillations of the PDF in generic Gaussian states was studied in [38, 39, 43]). The asymptotics of the photon distribution function (5.1) in the long-time limit are given in Appendix D.

\section{Conclusion}

The main results of the paper are as follows. We studied the behavior of the electromagnetic field quadrature variances in a one-dimensional cavity with resonantly oscillating ideal boundaries in the whole time interval $0 \leq t<\infty$ for all field modes and for any (small) value of the detuning parameter. We have shown that each field mode goes to a mixed quantum state due to the intermode interaction (caused by Doppler's effect on the moving mirrors). We found that squeezing can be observed only in the 'principal' modes with numbers $p(k+1 / 2)$, where integer $p$ is close to the ratio of the wall vibration frequency to the frequency of the fundamental cavity mode. Analyzing the influence of the initial nonvacuum state of the field we discovered that the initial thermal fluctuations do not affect the minimal value of the quadrature variance (which is less than 1/2) in the long-time limit. This result is important from the practical point of view, since it shows that certain significant features of the Nonstationary Casimir Effect are not sensitive to the temperature (see also [23] in the case of the three-dimensional cavity). We found the photon distribution functions $f_{m}(n)$ for all modes. For the modes which do not exhibit squeezing, the PDF coincides with a time-dependent Planck's distribution, while the PDF in the distinct 'principal' modes differs from Planck's one, being more 'flat' for $n \gg 1$.

\section{Acknowledgement}

MAA thanks Brazilian agency CNPq for the support (project 110524/97-7). 
Squeezing and photon distribution in a vibrating cavity

\section{Appendix A. Some properties of the Bogoliubov coefficients $\rho_{m}^{(n)}$}

The non-zero coefficients $\rho_{\mu}^{(n)}$ for the 'principal' modes read

$$
\begin{aligned}
\rho_{p m+p / 2}^{(p n+p / 2)} & =\frac{\Gamma(n+3 / 2) \kappa^{n-m} \lambda^{m+n+1}}{\Gamma(m+3 / 2) \Gamma(1+n-m)} \\
& \times F\left(n+1 / 2,-m-1 / 2 ; 1+n-m ; \kappa^{2}\right), \quad n \geq m \\
\rho_{p m+p / 2}^{(p n+p / 2)} & =\frac{(-1)^{m-n} \Gamma(m+1 / 2) \kappa^{m-n} \lambda^{m+n+1}}{\Gamma(n+1 / 2) \Gamma(1+m-n)} \\
& \times F\left(m+1 / 2,-n-1 / 2 ; 1+m-n ; \kappa^{2}\right), \quad m \geq n \\
\rho_{-p m-p / 2}^{(p n+p / 2)} & =\frac{(-1)^{m} \Gamma(m+1 / 2) \Gamma(n+3 / 2) \kappa^{n+m+1} \lambda^{n-m}}{\pi \Gamma(2+n+m)} \\
& \times F\left(n+1 / 2, m+1 / 2 ; 2+n+m ; \kappa^{2}\right) .
\end{aligned}
$$

Using the formula 44

$$
F(a, b ; a+b+1 ; 1)=\frac{\Gamma(a+b+1)}{\Gamma(a+1) \Gamma(b+1)}
$$

one can find the asymptotics of the coefficients $\rho_{m}^{(n)}$ for $\kappa \rightarrow 1$ [17]

$$
\rho_{p m+j}^{(p n+j)}(\tau \gg 1) \approx \frac{\sin [\pi(m+j / p)]}{\pi(m+j / p)}(a+i \gamma)^{m+n+2 j / p} \sigma^{n-m}
$$

In particular, for $p=2$ and for the odd ('principal') modes

$$
\begin{aligned}
& \rho_{2 m+1}^{(2 n+1)}(\tau \gg 1) \approx \frac{2(-1)^{m}}{\pi(2 m+1)}(a+i \gamma)^{m+n+1}, \\
& \rho_{-2 m-1}^{(2 n+1)}(\tau \gg 1) \approx \frac{2(-1)^{m}}{\pi(2 m+1)}(a+i \gamma)^{n-m}
\end{aligned}
$$

It is known [45] that the hypergeometric function $F(a, b ; c ; z)$ with 'half-integral' parameters $a, b$ and an integral parameter $c$ can be expressed in terms of the complete elliptic integrals

$$
\begin{aligned}
& \mathbf{K}(\kappa)=\int_{0}^{\pi / 2} \frac{\mathrm{d} \alpha}{\sqrt{1-\kappa^{2} \sin ^{2} \alpha}}=\frac{\pi}{2} F\left(\frac{1}{2}, \frac{1}{2} ; 1 ; \kappa^{2}\right) \\
& \mathbf{E}(\kappa)=\int_{0}^{\pi / 2} \mathrm{~d} \alpha \sqrt{1-\kappa^{2} \sin ^{2} \alpha}=\frac{\pi}{2} F\left(-\frac{1}{2}, \frac{1}{2} ; 1 ; \kappa^{2}\right) .
\end{aligned}
$$

In particular,

$$
\begin{aligned}
& \rho_{1}^{(1)}=\frac{2 \lambda(\kappa)}{\pi} \mathbf{E}(\kappa), \quad \rho_{-1}^{(1)}=\frac{2}{\pi \kappa}\left[\mathbf{E}(\kappa)-\tilde{\kappa}^{2} \mathbf{K}(\kappa)\right] \\
& \rho_{3}^{(1)}=\frac{2 \lambda^{2}(\kappa)}{3 \pi \kappa}\left[\left(1-2 \kappa^{2}\right) \mathbf{E}(\kappa)-\tilde{\kappa}^{2} \mathbf{K}(\kappa)\right] \\
& \rho_{-3}^{(1)}=-\frac{2}{3 \pi \kappa^{2} \lambda(\kappa)}\left[\left(2-\kappa^{2}\right) \mathbf{E}(\kappa)-2 \tilde{\kappa}^{2} \mathbf{K}(\kappa)\right]
\end{aligned}
$$


where $\tilde{\kappa} \equiv \sqrt{1-\kappa^{2}}$ and $\lambda(\kappa)=\sqrt{1-\gamma^{2} \kappa^{2}}+i \gamma \kappa$.

The general structure of the coefficients $\rho_{\mu}^{(1)}$ (we confine ourselves to the case $p=2$ ) is as follows

$$
\begin{aligned}
& \rho_{2 m+1}^{(1)}=\frac{2 \lambda^{m+1}(\kappa)}{\pi \kappa^{m}}\left[f_{m}\left(\kappa^{2}\right) \mathbf{E}(\kappa)+\tilde{\kappa}^{2} g_{m}\left(\kappa^{2}\right) \mathbf{K}(\kappa)\right] \\
& \rho_{-2 m-1}^{(1)}=\frac{2}{\pi \kappa^{m+1} \lambda^{m}(\kappa)}\left[r_{m}\left(\kappa^{2}\right) \mathbf{E}(\kappa)+\tilde{\kappa}^{2} s_{m}\left(\kappa^{2}\right) \mathbf{K}(\kappa)\right]
\end{aligned}
$$

where $f_{m}(x), g_{m}(x), r_{m}(x), s_{m}(x)$ are the polynomials of the degree $m$ which can be found from the recurrence relations (2.6).

The Taylor expansions of the complete elliptic integrals at $\kappa \rightarrow 0$ (when $\kappa \approx a p \tau$ ) read

$$
\mathbf{E}(\kappa)=\frac{\pi}{2}\left(1-\frac{1}{4} \kappa^{2}-\frac{3}{64} \kappa^{4}+\cdots\right), \quad \mathbf{K}(\kappa)=\frac{\pi}{2}\left(1+\frac{1}{4} \kappa^{2}+\frac{9}{64} \kappa^{4}+\cdots\right)
$$

whereas their asymptotic behaviours at $\tilde{\kappa} \rightarrow 0$ are given by the formulae [4]

$$
\begin{aligned}
& \mathbf{K}(\kappa) \approx \ln \frac{4}{\tilde{\kappa}}+\frac{1}{4}\left(\ln \frac{4}{\tilde{\kappa}}-1\right) \tilde{\kappa}^{2}+\cdots \\
& \mathbf{E}(\kappa) \approx 1+\frac{1}{2}\left(\ln \frac{4}{\tilde{\kappa}}-\frac{1}{2}\right) \tilde{\kappa}^{2}+\cdots .
\end{aligned}
$$

In this case $\tilde{\kappa} \approx a / \sinh (a p \tau)$ and $\ln (1 / \tilde{\kappa}) \approx a p \tau$.

\section{Appendix B. Calculation of integrals}

To calculate, for instance, the variance $U_{1}$ we use equations $(3.7)$ and $(\mathrm{A.10})$ and replace the derivative over $\tau$ by the derivative with respect to $\kappa$ using the relation (if $p=2$ ) $\mathrm{d} \kappa=2 \beta \tilde{\kappa}^{2} \mathrm{~d} \tau$, where $\beta=\sqrt{1-\gamma^{2} \kappa^{2}}$. We arrive at the equation

$$
\begin{aligned}
\frac{\mathrm{d} U_{1}}{\mathrm{~d} \kappa}= & -\frac{2}{\pi^{2} \tilde{\kappa}^{2} \kappa^{2} \beta}\left\{\left[\kappa^{2}\left(1-2 \gamma^{2} \kappa^{2}\right)+1-2 \beta \kappa\right] \mathbf{E}^{2}(\kappa)\right. \\
& \left.-2 \tilde{\kappa}^{2}(1-\beta \kappa) \mathbf{E}(\kappa) \mathbf{K}(\kappa)+\tilde{\kappa}^{4} \mathbf{K}^{2}(\kappa)\right\} .
\end{aligned}
$$

Let us consider first the case $\gamma=0$, when $\beta=1$. Taking into account the differentiation rules 44

$$
\frac{\mathrm{d} \mathbf{K}(\kappa)}{\mathrm{d} \kappa}=\frac{\mathbf{E}(\kappa)}{\kappa \tilde{\kappa}^{2}}-\frac{\mathbf{K}(\kappa)}{\kappa}, \quad \frac{\mathrm{d} \mathbf{E}(\kappa)}{\mathrm{d} \kappa}=\frac{\mathbf{E}(\kappa)-\mathbf{K}(\kappa)}{\kappa}
$$

we may suppose that the factor $\tilde{\kappa}^{2}$ in the denominator of the right-hand side of equation (B.1) comes from the derivative $\mathrm{d} \mathbf{K} / \mathrm{d} \kappa$. Thus it is natural to look for the solution in the form

$$
U_{1}=\frac{2}{\pi^{2} \kappa}\left[A(\kappa) \mathbf{K}^{2}(\kappa)+B(\kappa) \mathbf{K}(\kappa) \mathbf{E}(\kappa)+C(\kappa) \mathbf{E}^{2}(\kappa)\right],
$$

where $A(\kappa), B(\kappa)$ and $C(\kappa)$ are some polynomials of $\kappa$. Putting the expression (B.3) into equation $(\mathbb{B} .1)$ we obtain a set of coupled equations for the unknown functions $A, B, C$. Writing $A(\kappa)=a_{0}+A_{1}(\kappa), B(\kappa)=b_{0}+B_{1}(\kappa), C(\kappa)=c_{0}+C_{1}(\kappa)$ we determine the constant coefficients $a_{0}, b_{0}$ and $c_{0}$ by putting $\kappa=0$ in that equations. 
Then we obtain new equations for the functions $A_{1}(\kappa), B_{1}(\kappa)$ and $C_{1}(\kappa)$ and repeat the procedure. After a few steps we arrive at the equations which have obvious trivial solutions $A_{n}=B_{n}=C_{n}=0$. This confirms our hypothesis on the polynomial structure of the functions $A(\kappa), B(\kappa)$ and $C(\kappa)$ and gives the final answer. The equations for the variances $U_{\mu}, V_{\mu}$, etc. with $\mu \geq 3$ can be integrated in the same manner, the only difference is that one should write $\kappa^{\mu}$ instead of $\kappa$ in the denominator of the expression like (B.3). In the generic case $\gamma \neq 0$ we notice that the factor $\beta$ can appear in the denominator of the expression (B.1) as a result of differentiating the function $\beta(\kappa)$, since $\mathrm{d} \beta / \mathrm{d} \kappa=-\gamma^{2} \kappa / \beta$. Therefore we split each function, $A, B, C$ in the ' $\beta$-even' and ' $\beta$-odd' parts like $A=A_{e}(\kappa)+\beta(\kappa) A_{o}(\kappa)$. The equations for the 'even' and 'odd' coefficients turn out independent, and we solve them using the procedure described above. The equation (3.21) was integrated using the same scheme.

\section{Appendix C. Asymptotics of the minimal variance and purity factor}

For the initial diagonal density matrix (in the Fock basis), combining the equations (3.11)-(3.13) and (4.5), we write the variances at $\tau \gg 1$ as (we omit the subscript $\mu$ )

$$
\left(\begin{array}{l}
U(\tau) \\
V(\tau) \\
Y(\tau)
\end{array}\right)=\left(\begin{array}{l}
2 F \sin ^{2}(\chi / 2)+f \\
2 F \cos ^{2}(\chi / 2)+g \\
-F \sin \chi+h
\end{array}\right), \quad F=\frac{8(a \tau+\mathcal{Z})}{\pi^{2} \mu}, \quad \chi=\mu \phi .
$$

The functions $f, g$ and $h$ are much smaller than $F$. At $\tau \rightarrow \infty$ these functions tend to finite limits which do not depend on the initial state, since they can be found by integrating equations (3.11)-(3.13). Thus we have $U+V=2 F+f+g$, whereas

$$
(U-V)^{2}+4 Y^{2}=4 F^{2}+4 F[(g-f) \cos \chi-2 h \sin \chi]+(f-g)^{2}+4 h^{2} .
$$

For $F \gg f, g, h$ we have

$$
\sqrt{(U-V)^{2}+4 Y^{2}}=2 F+(g-f) \cos \chi-2 h \sin \chi+\mathcal{O}(1 / F)
$$

so the minimal variance $u(\tau)(3.15)$ tends to the finite limit

$$
u(\infty)=f \cos ^{2}(\chi / 2)+g \sin ^{2}(\chi / 2)+h \sin \chi
$$

which does not depend on $\mathcal{Z}$, i.e. on the initial state.

Analogously, $U V-Y^{2}=2 F u(\infty)+\mathcal{O}(1) \sim \tau$ for $\tau \gg 1$. Consequently, the purity factor $\chi$ asymptotically goes to zero as $\tau^{-1 / 2}$.

\section{Appendix D. Photon distribution in the Gaussian state}

In the most compact form the information on the photon distribution $f(n)$ in some mode (we suppress here the mode index) is contained in the generating function

$$
G(z)=\sum_{n=0}^{\infty} f(n) z^{n}
$$


For the most general Gaussian state it was given in [38, 39] (for a single mode):

$$
G(z)=[\mathcal{G}(z)]^{-1 / 2} \exp \left(\frac{1}{D}\left[\frac{z g_{1}-z^{2} g_{2}}{\mathcal{G}(z)}-g_{0}\right]\right)
$$

where

$$
\begin{aligned}
\mathcal{G}(z)= & \frac{1}{4}\left[(1+z)^{2}+4\left(U V-Y^{2}\right)(1-z)^{2}+2(U+V)\left(1-z^{2}\right)\right] \\
D= & 1+2(U+V)+4\left(U V-Y^{2}\right)=4 \mathcal{G}(0) \\
g_{0}= & \langle\hat{p}\rangle^{2}(2 U+1)+\langle\hat{q}\rangle^{2}(2 V+1)-4\langle\hat{p}\rangle\langle\hat{q}\rangle Y \\
g_{1}= & 2\langle\hat{p}\rangle^{2}\left(U^{2}+Y^{2}+U+\frac{1}{4}\right)+2\langle\hat{q}\rangle^{2}\left(V^{2}+Y^{2}+V+\frac{1}{4}\right) \\
& -4\langle\hat{p}\rangle\langle\hat{q}\rangle Y(U+V+1) \\
g_{2}= & 2\langle\hat{p}\rangle^{2}\left(U^{2}+Y^{2}-\frac{1}{4}\right)+2\langle\hat{q}\rangle^{2}\left(V^{2}+Y^{2}-\frac{1}{4}\right)-4\langle\hat{p}\rangle\langle\hat{q}\rangle Y(U+V)
\end{aligned}
$$

If $\langle\hat{p}\rangle=\langle\hat{q}\rangle=0$, then the probability $f(n)$ is expressed in terms of the Legendre polynomials: see equation (5.1). In the generic case $f(n)$ is related to the twodimensional 'diagonal' Hermite polynomials [38]:

$$
f(n)=\frac{\mathcal{F}_{0}}{n !} H_{n n}^{\{\mathcal{R}\}}\left(x, x^{*}\right)
$$

where

$$
\begin{aligned}
& \mathcal{F}_{0}=f(0)=2 D^{-1 / 2} \exp \left(-g_{0} / D\right) \\
& x=\frac{\sqrt{2}\{(2 V-1)\langle\hat{q}\rangle-2 Y\langle\hat{p}\rangle+i[(1-2 U)\langle\hat{p}\rangle+2 Y\langle\hat{q}\rangle]\}}{2(U+V)-4\left(U V-Y^{2}\right)-1}
\end{aligned}
$$

and $2 \times 2$ symmetric matrix $\mathcal{R}$ has the elements

$$
\mathcal{R}_{11}=\mathcal{R}_{22}^{*}=\frac{2}{D}(V-U-2 i Y), \quad \mathcal{R}_{12}=\mathcal{R}_{21}=\frac{1}{D}\left[1-4\left(U V-Y^{2}\right)\right]
$$

The two-dimensional Hermite polynomials are defined via the expansion [46]

$$
\exp \left(-\frac{1}{2} \mathbf{a} \mathcal{R} \mathbf{a}+\mathbf{a} \mathcal{R} \mathbf{x}\right)=\sum_{m, n=0}^{\infty} \frac{a_{1}^{m} a_{2}^{n}}{m ! n !} H_{m n}^{\{\mathcal{R}\}}\left(x_{1}, x_{2}\right)
$$

where $\mathbf{x}=\left(x_{1}, x_{2}\right), \mathbf{a}=\left(a_{1}, a_{2}\right)$. The properties of these polynomials were studied recently in [39, 43]. In particular, they can be expressed as finite sums of the products of the usual (one-dimensional) Hermite polynomials. The corresponding formula for the probabilities reads 38

$$
f(n)=\mathcal{F}_{0}\left(\frac{\Delta}{D}\right)^{n} \sum_{k=0}^{n}\left(\frac{S}{\Delta}\right)^{k} \frac{n !}{[(n-k) !]^{2} k !}\left|H_{n-k}(\xi)\right|^{2}
$$

where

$$
\Delta=\sqrt{(U-V)^{2}+4 Y^{2}}, \quad S=4\left(U V-Y^{2}\right)-1
$$




$$
\xi=\frac{(2 V+1)\langle\hat{q}\rangle-2 Y\langle\hat{p}\rangle+i[(1+2 U)\langle\hat{p}\rangle-2 Y\langle\hat{q}\rangle]}{[2 D(V-U-2 i Y)]^{1 / 2}}
$$

The photon distribution function (5.1) can be simplified in the long-time limit $\tau \gg 1$, when the average number of created photons $\mathcal{N} \equiv \bar{n} \approx(V+U) / 2$ exceeds 1. Then the mean-square fluctuation of the photon number has the same order of magnitude as the mean photon number itself, $\sqrt{\sigma_{n}} \approx \sqrt{2} \mathcal{N}$, and the most significant part of the spectrum corresponds to the values $n \gg 1$. Using the Laplace-Heine asymptotical formula for the Legendre polynomial [47]

$$
P_{n}(z) \approx \frac{\left(z+\sqrt{z^{2}-1}\right)^{n+1 / 2}}{\sqrt{2 \pi n}\left(z^{2}-1\right)^{1 / 4}}, \quad n \gg 1
$$

one can simplify (5.1) for the fixed values of the invariant variances $u$ and $v$ as

$$
f(n) \approx \frac{1}{\sqrt{\pi n(v-u)}}\left(\frac{2 v-1}{2 v+1}\right)^{n+1 / 2}
$$

provided the positive difference $v-u$ is not too small. Another approximate formula can be used if $v \gg 1$ but $u \sim 1$ :

$$
f(n) \approx \frac{\sqrt{2}(2 u-1)^{n / 2}}{\sqrt{v}(2 u+1)^{(n+1) / 2}} e^{-n /(2 v)} P_{n}\left(\frac{2 u}{\sqrt{4 u^{2}-1}}\right), \quad n \ll 8 v^{2} .
$$




\section{References}

[1] Dodonov V V, Klimov A B and Man'ko V I 1989 Phys. Lett. A 142511

[2] Schwinger J 1993 Proc. Natl. Acad. Sci. USA 90 958, 2105, 4505, 7285

[3] Barton G and Eberlein C 1993 Ann. Phys. (NY) 227222

[4] Lambrecht A, Jaekel M-T and Reynaud S 1996 Phys. Rev. Lett. 77615

[5] Dodonov V V and Klimov A B 1996 Phys. Rev. A 532664

[6] Johnston H and Sarkar S 1996 J. Phys. A: Math. Gen. 291741

[7] Mundarain D F and Maia Neto P A 1998 Phys. Rev. A 571379

[8] Dalvit D A R and Mazzitelli F D 1998 Phys. Rev. A 572113

[9] Schützhold R, Plunien G and Soff G 1998 Phys. Rev. A 572311

[10] Janowicz M 1998 Phys. Rev. A 574784

[11] Ji J-Y, Jung H-H and Soh K-S 1998 Phys. Rev. A 574952

[12] Ji J-Y, Soh K-S, Cai R-G and Kim S P 1998 J. Phys. A: Math. Gen. 31 L457

[13] Lambrecht A, Jaekel M-T and Reynaud S 1998 Eur. Phys. J. D 395

[14] Golestanian R and Kardar M 1998 Phys. Rev. A 581713

[15] Dodonov V V 1998 Phys. Rev. A 584147

[16] Pendry J B 1998 J. Mod. Opt. 452389

[17] Dodonov V V 1998 J. Phys. A: Math. Gen. 319835

[18] Mendonça J P F, Maia Neto P A and Takakura F I 1999 Opt. Comm. 160335

[19] Wu Y, Chan K W, Chu M-C and Leung P T 1999 Phys. Rev. A 591662

[20] Miri F and Golestanian R 1999 Phys. Rev. A 592291

[21] Wu Y, Chu M-C and Leung P T 1999 Phys. Rev. A 593032

[22] Dalvit D A R and Mazzitelli F D 1999 Phys. Rev. A 593049

[23] Plunien G, Schützhold R and Soff G 1999 Los Alamos archive preprint quant-ph/9906122

[24] Cooper J 1993 IEEE Trans. Antennas Prop. 411365

[25] Dittrich J, Duclos P and Gonzalez N 1998 Rev. Math. Phys. 10925

[26] Dodonov V V, Klimov A B and Man'ko V I 1990 Phys. Lett. A 149225

[27] Chizhov A V, Schrade G and Zubairy M S 1997 Phys. Lett. A 230269

[28] Dodonov V V and Klimov A B 1992 Phys. Lett. A 167309

[29] Dodonov V V, Klimov A B and Nikonov D E 1993 J. Math. Phys. 342742

[30] Moore G T 1970 J. Math. Phys. 112679

[31] Law C K 1994 Phys. Rev. A 49433 1995 Phys. Rev. A 512537

[32] Dodonov V V, Kurmyshev E V and Man'ko V I 1980 Phys. Lett. A 79150

[33] Dodonov V V, Klimov A B and Man'ko V I 1993 Squeezed and Correlated States of Quantum Systems (Proc. Lebedev Physics Institute 205) ed M A Markov (Commack: Nova Science) p 61

[34] Dodonov V V, Man'ko V I and Polynkin P G 1994 Phys. Lett. A 188232

[35] Dodonov V V and Man'ko V I 1987 Group Theory, Gravitation and Elementary Particle Physics (Proc. Lebedev Physics Institute 167) ed A A Komar (Commack: Nova Science) p 7

[36] Dodonov V V, Malkin I A and Man'ko V I 1975 J. Phys. A: Math. Gen. 8 L19 1975 Int. J. Theor. Phys. 1437

[37] Dodonov V V and Man'ko V I 1989 Invariants and the Evolution of Non-Stationary Quantum Systems (Proc. Lebedev Physics Institute 183) (Commack: Nova Science) p 263

[38] Dodonov V V, Man'ko O V and Man'ko V I 1994 Phys. Rev. A 49 2993; 50813

[39] Dodonov V V and Man'ko V I 1994 J. Math. Phys. 354277

[40] Vourdas A and Weiner R M 1987 Phys. Rev. A 365866

[41] Schleich W and J.A.Wheeler J A 1987 J.Opt.Soc.Am. B 41715

[42] Dodonov V V, Klimov A B and Man'ko V I 1989 Phys. Lett. A 134211

[43] Dodonov V V 1994 J. Phys. A: Math. Gen. 276191

[44] Gradshtein I S and Ryzhik I M 1994 Tables of Integrals, Series and Products (New York: Academic) 
[45] Prudnikov A P, Brychkov Yu A and Marichev O I 1986 Integrals and Series. Additional Chapters (Moscow: Nauka)

[46] Erdélyi A (ed) 1953 Bateman Manuscript Project, Higher Transcendental Functions (New York: McGraw-Hill)

[47] Szegö G 1959 Orthogonal Polynomials (New York: American Mathematical Society) 


\section{Figure captions}

Figure 1. The minimal variance $u_{1}$, the maximal variance $v_{1}$, the purity factor $\chi$, the mean photon number $\mathcal{N}_{1}$ and Mandel's parameter $Q$ of the fundamental mode $\mu=1$ versus the universal parameter $\kappa(2.8)$.

Figure 2. The photon distribution function in the fundamental field mode $\mu=1$ for $\tau=5$ and $\gamma=0, p=2$ (points connected with solid lines). Points connected with dashed lines correspond to the Planck distribution with the same mean photon number. 


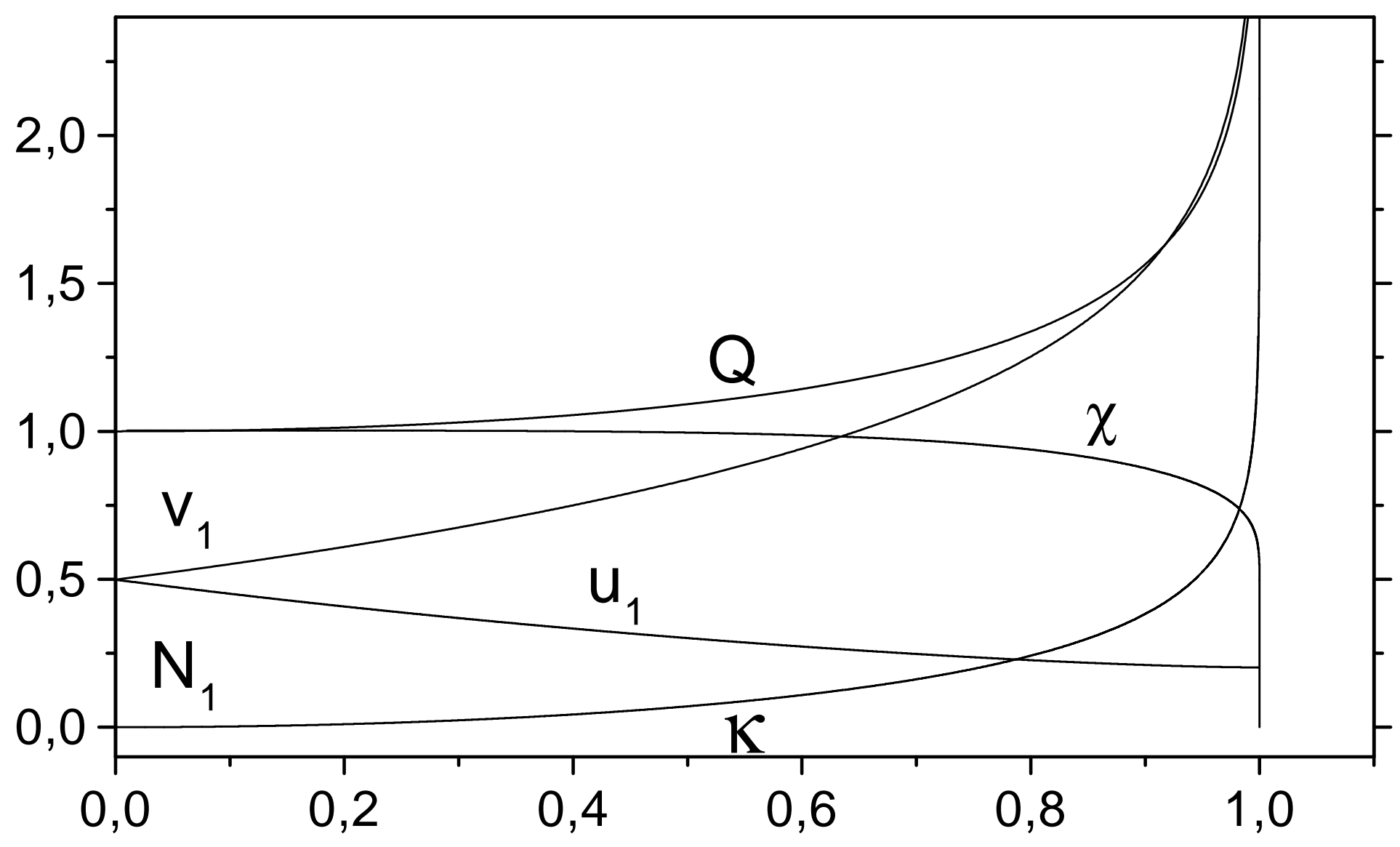




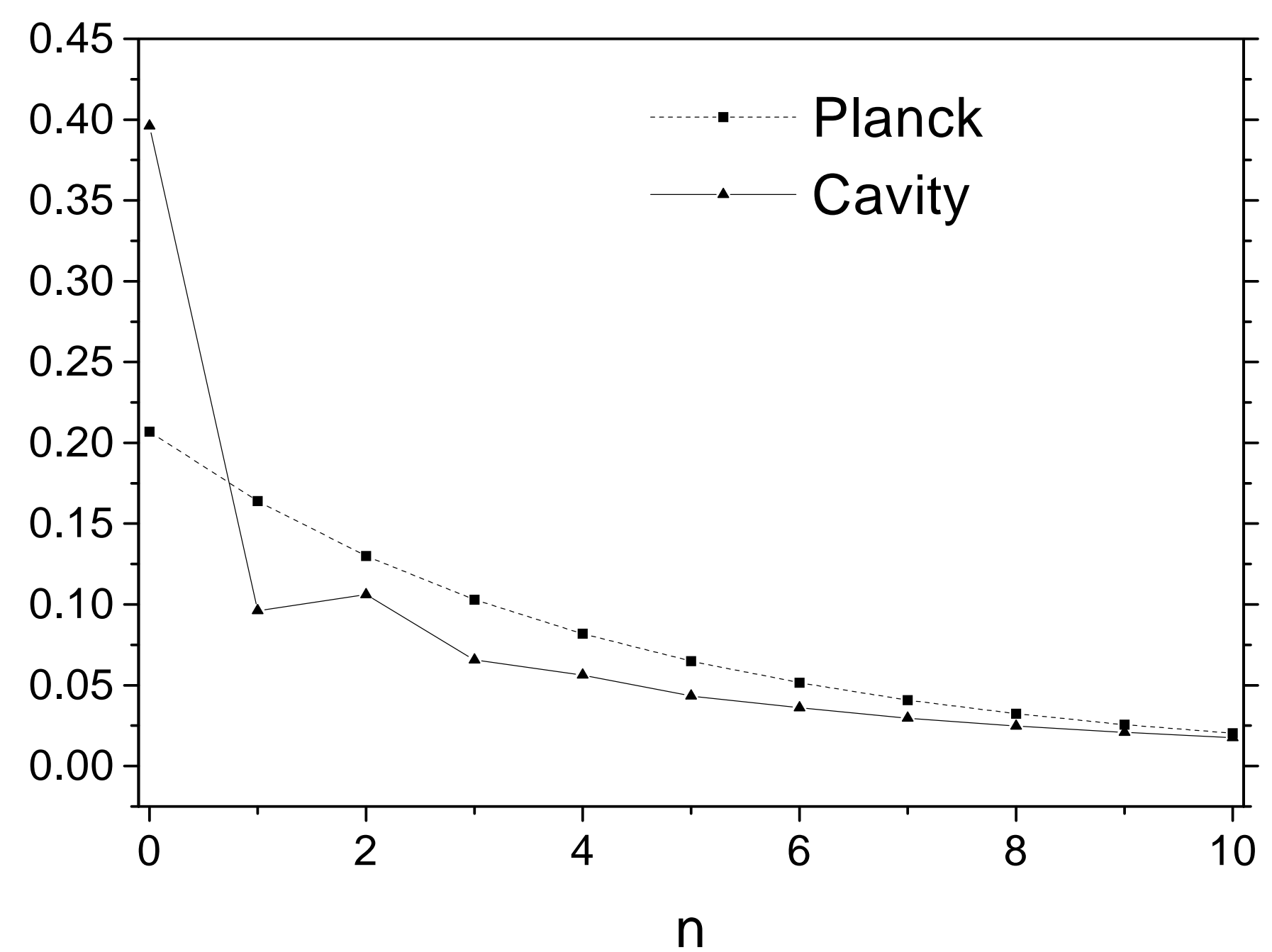

